\title{
Study on the NN Decoupling Control System of Air-cushioned Headbox
}

\author{
Zhongjun Xiao \\ College of Electric and Information Engineering, Shaanxi University of Science \& Technology, Xi' an 710021, China \\ Tel: 86-29-8616-8631 E-mail: xiaodongjun@sust.edu.cn
}

Mengxiao Wang

Xi Wei Measurement and Control Corporation, Xianyang 712081, China

Tel: 86-29-3357-7920Ｅ-mail: qywjs@163.com

\begin{abstract}
The headbox is the key hinge to link the pulp supply system with the sheet forming in the papermaking process. The primary parameters include the total pressure and the stock level which couple each other in the box, and they decide the distribution of the web cross-directional basis weight and influence the paper forming quality. Here taking widely used air-cushion type headbox as the plant, a new neural network (NN) decoupling control system is proposed to overcome the coupling relation between the total pressure and the stock level, decrease the adjusting time as well as to achieve robustness, fault-tolerance and the self-study ability in different environments. The practice has proved that the headbox control system based on $\mathrm{NN}$ decoupling algorithm could satisfy the industrial control requirements in the papermaking process well.
\end{abstract}

Keywords: Papermaking process, Air-cushioned headbox, Neural network (NN), Decoupling control

\section{Introduction}

The regularity distribution and the sheet quality of the finished paper are decided by the dispersion degree of the fiber in the headbox and the regularity of the jetting pulp in the stock inlet of the headbox, so the design and operation of the headbox become into the key issues in the papermaking process (Tang, 2005, P.129-134). In the work process of the air-cushioned headbox, the main control parameters include the total pressure and the stock level. The intention to control the total pressure is to acquire the even pulp flow velocity and flux jetting from the headbox to the net. The intention to control the stock level is to acquire proper pulp flow area to reduce the changes of crosscurrent and concentration, and produce the controllable overfall to limit the agglutination of the fibers. But the total pressure and the stock level couples each other, at present, to overcome the coupling between the total pressure and the stock level, many control systems such as the fuzzy control, NN decoupling control and self-adaptive control to quickly and exactly control the total pressure and the stock level (Tang, 2006, P.107-114).

According to the characteristics of the headbox and the papermaking process, the NN control algorithm is adopted to exactly control the total pressure and the stock level of the headbox and ensure the web cross-directional basis weight and the quality of the moisture measurement.

$\mathrm{NN}$ is the complex network linked by large numbers of nerve cells each other. It could simulate the distributed work characters and the self-organized function of human brain cell, and it possesses very strong abilities such as parallel processing, self-adaptability, self-study and nonlinear mapping, and it can be adapted for the highly uncertain process control system and the nonlinear process control system.

\section{Control flow of air-cushioned type headbox}

Figure 1 can be used to describe the fourdrinier wet flow of the headbox automatic adjustment system. The stocks from the flow system enter into the headbox through the control valve, and the headbox jets the stocks to the net according to acquired flux and velocity, and the wet sheets are formed through the net dehydration (Wang, 2003).

Figure 1 is the sketch map of the air-cushioned headbox system, and in the figure, $F W$ is the water jetting capacity of the headbox, $F A I$ is the air-cushioned air inflow of the headbox, $F V$ is the spillway discharge of the headbox, $C O$ is the 
stock outflow concentration of the headbox, and $F R$ is the stock inflow capacity of the headbox. In the air-cushioned headbox, the following formula exists between the stock jetting velocity $V$ and the total pressure of the headbox $P$.

$V=\mu \sqrt{2 g \cdot P}$

In the formula, $\mu$ is the coefficient about the material property and the form of the head box, and the total pressure $P$ includes the air-cushioned pressure $\left(P_{\text {air }}\right)$ and the static pressure of the stock level $\left(H_{\text {stock }}\right)$. Therefore, the stock level or the air-cushioned pressure or the total pressure all can be adjusted to adjust the stock jetting velocity. In the total pressure and the stock level, the key is to stabilize the total pressure in order to stabilize the stock velocity. The intention to control the stock level is only to keep the needed flow character in the transmission process of the head box, and under the premise that the total pressure doesn't change, the fluctuation of the stock level in small range is allowed.

So the control application project seen in Figure 2 is proposed. The control of the total pressure is to change the stock adjustment valve by the total pressure controller (PC), i.e. adjusting the stock inflow capacity. The control of the stock level is actualized by the stock level controller (LC) which adjusts the air-cushioned outflow capacity, and the cascade control system composed by the ratio between the stock velocity and the net velocity and the total pressure is added. This control method can not only control the total pressure and the stock level, but can control the ratio between the stock velocity and the net velocity. The emphasis of the control is the adjusted parameter, i.e. the total pressure, and the adjustment capacity is the stock inflow capacity. Therefore, when the adjustment system controls the total pressure well, the flux of the headbox can be stabilized, and the stock inflow capacity must balance with the outflow capacity.

The intention of the headbox control is to acquire stable and even flux and stock jetting velocity of the headbox, and both are always decided by the total pressure when the inlet degree is certain, so the total pressure is the most important and strict parameter in the headbox control. The proper stock level change will not obviously influence the stability and the regularity of the flux of the headbox, i.e. the stock level is the secondary control parameter in the headbox control. Because of the coupling function of the total pressure and the stock level, the general adjustment system can hardly control them in the optimal state simultaneously, so to achieve the control objective of the headbox and the control the total pressure in the optimal state, the stock level should be allowed to change little. As viewed form the stock balance, only the inflow stock capacity and the outflow stock capacity of the headbox achieve balanced, the flux of the headbox can return to the stable state. In other words, the key of the headbox automatic adjustment is to exactly adjust the stock capacity flowing into the headbox and make the capacity equal to the appointed flux of the headbox.

\section{NN decoupling control system of air-cushioned headbox}

\subsection{Coupling characters of total pressure and stock level}

In the control process of the total pressure and the stock level in the headbox, when any one of the values which control the stock inlet capacity and the air-cushioned flux acts, both the total pressure and the stock level will change. In one experiment, put the value which controls the air-cushioned air flux on the manual state, and change the stock inlet capacity, when the total pressure ascends from $15.2 \mathrm{KPa}$ to $15.96 \mathrm{KPa}$, the stock level ascends from $27.0 \mathrm{~cm}$ to $33.9 \mathrm{~cm}$, which indicates that the total pressure is associated and coupled with the stock level control loop. If the decoupling control is not implemented, the total pressure and the stock level can not achieve the optimal state simultaneously, which will make the character of the net sheet be influenced, and influence the papermaking quality of the paper. Therefore, to achieve optimal papermaking condition, it is necessary to implement decoupling control of the total pressure and the stock level in the headbox.

\subsection{Characteristics of $N N$ decoupler}

There are strong coupling phenomena in many multi-variable control systems, and if certain decoupling measure is not adopted, the system can not acquire satisfactory control effect, even can not be controlled. In many situations, the decoupling equipments are very complex, and even they are not implemented in the physical practice. In addition, to implement decoupling, the mathematical model of the controlled process must be known in advance, and even if that can be done, the work of the system will not be ideal because of the nicety of the model, the nonlinearity and the time-varying of the system. NN possesses the self-study ability and the nonlinear mapping ability, so it can solve above problems well.

The decoupling of the system is divided into the static decoupling and the dynamic decoupling. In practice, the static decoupling is generally adopted, because the compensation designed by the dynamic decoupling can not be implemented, or can be implemented too complexly. When the system has time-varying character or obvious nonlinearity, it is impossible to realize the static decoupling, because the decoupling stage is required to possess the self-adaptive ability. As a new nonlinear system description method, $\mathrm{NN}$ can realize complex function by the composite of simple nonlinear functions. The trainability of $\mathrm{NN}$ and the commonality of the structure make NN possess self-adaptive ability to fulfill the requirement of decoupling. Therefore, if $\mathrm{NN}$ is used in locale decoupling, it can overcome the nonlinearity and the time-varying character of the system well, break past unchangeable decoupling mode 
and enhance the decoupling precision of the system, so $\mathrm{NN}$ will the development direction in the future decoupling of control process as the decoupler.

\subsection{Headbox NN decoupling control project}

In the total pressure and stock level control system, according to the characteristics of $\mathrm{NN}$, the feed forward $\mathrm{NN}$ is selected to develop and study as the decoupler.

Different connections between NN and controlled objective can compose different NN decoupling compensation projects. In the actual system, the pressure adjustment system stabilizes the total pressure by the stock inlet capacity adjusted by the stock pump, and the stock level adjustment system stabilizes the stock level by the electric conduction adjuster controlling the air-cushioned air flux. The decoupler is put in front of the controller or in the feedback loop. In the article, the decoupler is put in front of the controller. For the paired input and output system of the headbox, the control frame chart is seen in Figure 3.

\section{NN decoupling controller}

\subsection{NN decoupling algorithm}

The indexes adopted by the NN decoupling algorithm include the generalized objective Bristol first coefficient matrix containing the NN compensation, i.e. each sensitivity matrix of controlled variable about the deviation, and here, the paired input and output system is discussed as follows (Shen, 2004, P.151-156).

$\mathbf{B}=\left[\frac{\partial Y}{\partial E}\right]=\frac{\partial\left(y_{1}, y_{2}\right)}{\partial\left(e_{1}, e_{2}\right)}=\left[\begin{array}{ll}\frac{\partial y_{1}}{\partial e_{1}} & \frac{\partial y_{1}}{\partial e_{2}} \\ \frac{\partial y_{2}}{\partial e_{1}} & \frac{\partial y_{2}}{\partial e_{2}}\end{array}\right]$

If the generalized objective is decoupling, so the matrix B can be turned into the diagonal matrix through the line or row transformation.

The decoupling train objective is

$$
\min _{W} \mathrm{E}=\frac{1}{2} \sum_{i=1}^{2}\left[B_{i}^{[s]}-\frac{\partial y_{i}(W)}{\partial \mathrm{E}_{i}}\right]^{2}+\frac{1}{2} \sum_{\substack{i=1 \\ i \neq j}}^{2}\left[\frac{\partial y_{i}(W)}{\partial \mathrm{E}_{j}}\right]^{2}
$$

In above formula, $B_{i}^{[s]}$ is the generalized objective plus of the decoupling system including the plus of the adjuster, and $W$ is the training parameter of $\mathrm{NN}$.

The training algorithm of NN adopts the grads algorithm.

$\Delta W=-\eta \frac{\partial E(W)}{\partial W}$

$\eta$ is the learning step length, and when $\eta$ is small enough, the enough more reasonable interior units should be selected, and the function could make $E(W)$ is differentiable for $W$, and the grades algorithm is always convergent.

Note $K_{i j}^{Q}$ is the static plus of the system stage $Q$ between the i'th input and the j'th input, and $Q$ can be any one of $R$ (controller), $P$ (controlled object) or $N(\mathrm{NN})$. From (1), (2) and (3),

$$
\begin{aligned}
& \frac{\partial E(W)}{\partial W}=\sum_{i=1}^{2}\left[B_{i}^{[s]}-\frac{\partial}{\partial e_{i}} y_{i}(W)\right] \frac{\partial^{2} y_{i}(W)}{\partial W \partial e_{i}} \\
& +\sum_{\substack{i=1 \\
i \neq j}}^{2}\left(\frac{\partial}{\partial e_{j}} y_{i}(W)\right) \frac{\partial^{2} y_{i}(W)}{\partial W \partial e_{j}} \\
& \frac{\partial y_{i}(W)}{\partial e_{i}}=\frac{\partial y_{i}(W)}{\partial u_{i}(W)} \frac{\partial u_{i}(W)}{\partial L_{i}(W)} \frac{\partial L_{i}(W)}{\partial e_{i}}=K_{i i}^{P} K_{i i}^{R} K_{i i}^{N}(W) \\
& \frac{\partial y_{i}(W)}{\partial e_{j}}=\frac{\partial y_{i}(W)}{\partial u_{i}(W)} \frac{\partial u_{i}(W)}{\partial L_{i}(W)} \frac{\partial L_{i}(W)}{\partial e_{j}} \\
& =K_{i i}^{P} K_{i i}^{R} K_{i j}^{N}(W)
\end{aligned}
$$

So,

$$
\begin{aligned}
& \frac{\partial^{2} y_{i}(W)}{\partial W \partial e_{i}}=\frac{\partial}{\partial W}\left[K_{i i}^{P} K_{i i}^{R} K_{i i}^{N}(W)\right] \\
& =K_{i i}^{P} K_{i i}^{R} \frac{\partial K_{i i}^{N}(W)}{\partial W}=K_{i i}^{P} K_{i i}^{R} T_{i i}(W)
\end{aligned}
$$




$$
\begin{aligned}
& \frac{\partial^{2} y_{i}(W)}{\partial W \partial e_{j}}=\frac{\partial}{\partial W}\left[K_{i i}^{P} K_{i i}^{R} K_{i j}^{N}(W)\right] \\
& =K_{i i}^{P} K_{i i}^{R} \frac{\partial K_{i j}^{N}(W)}{\partial W}=K_{i i}^{P} K_{i i}^{R} T_{i j}(W)
\end{aligned}
$$

Unite (7) and (8),

$$
\begin{aligned}
& \frac{\partial E(W)}{\partial W}=\sum_{i=1}^{2}\left[B_{i}^{[s]}-K_{i i}^{P} K_{i i}^{R} K_{i i}^{N}(W)\right] K_{i i}^{P} K_{i i}^{R} T_{i i}(W) \\
& +\sum_{\substack{i=1 \\
i \neq j}}^{2}\left(K_{i i}^{P} K_{i i}^{R} K_{i j}^{N}(W)\right) K_{i i}^{P} K_{i i}^{R} T_{i j}(W)
\end{aligned}
$$

If the decoupler form is known, the above algorithm can be used to acquire the structure of NN decoupler.

\subsection{NN decoupling compensator design}

The decoupler adopts the multi-layer feed forward NN (Li, 2007, P.107-114). Because the control system has the paired input and out structure, so there are 2 input and output nodes in the network. The quantity of the hidden node should exceed the quantity of input node, and in the simulation, the system will find the proper network node quantity.

First, set up a few nodes to train the network, and test the approaching error of the network, and then gradually increase the quantity of node until the test error doesn't decrease obviously. If the node quantity is too more when above method is adopted, two hidden layers can be adopted, and the total quantity of the node in two hidden layers usually is less than the quantity of the node in one hidden layer.

In formula (9), $K_{i i}^{R}$ and $K_{i i}^{P}$ respectively are the static pluses between the $\mathrm{i}$ 'th input and the $\mathrm{j}$ 'th input of of $R$ (controller) and $P$ (controlled object). $\quad K_{i i}^{R}$ can be obtained directly from the controller, and $K_{i i}^{P}$ can be measured by the experiment. In the feed forward three-layer NN, the input functions of various layers all adopt the logsig function (Zhong, 2007), and the network form is seen in Figure 4.

The weight matrixes of three layers respectively are $\mathbf{W}_{1}(2 \times 2), \mathbf{W}_{2}(S \times 2)$ and $\mathbf{W}_{3}(2 \times S)$. Suppose the inputs of three layers respectively are $\mathbf{O}_{1}, \mathbf{O}_{2}$ and $\mathbf{L}$, the inputs of three layers respectively are $\mathbf{X}_{1}, \mathbf{X}_{2}$ and $\mathbf{X}_{3}$.

$$
\begin{aligned}
& \mathbf{X}_{1}=\left[\begin{array}{l}
X_{1}(1) \\
X_{1}(2)
\end{array}\right], \quad \mathbf{x}_{2}=\left[\begin{array}{c}
X_{2}(1) \\
\vdots \\
X_{2}(S)
\end{array}\right], \quad \mathbf{X}_{3}=\left[\begin{array}{c}
X(1) \\
X(2)
\end{array}\right] \\
& \mathbf{O}_{1}=\left[\begin{array}{c}
O_{1}(1) \\
O_{1}(2)
\end{array}\right], \quad \mathbf{O}_{2}=\left[\begin{array}{c}
O_{2}(1) \\
\vdots \\
O_{2}(S)
\end{array}\right], \quad \mathbf{L}=\left[\begin{array}{c}
L(1) \\
L(2)
\end{array}\right]
\end{aligned}
$$

From the analysis,

$$
\begin{array}{lr}
\mathbf{X}_{1}(j)=\sum_{i=1}^{2} e_{i} W_{1}(j, i) & (j=1,2) \\
\mathbf{X}_{2}(j)=\sum_{i=1}^{2} O_{1}(i) W_{2}(j, i) & (j=1, \cdots, S) \\
\mathbf{X}_{3}(j)=\sum_{i=1}^{S} O_{2}(i) W_{3}(j, i) & (j=1,2) \\
\mathbf{O}_{1}(j)=f\left(X_{1}(j)\right) & (j=1,2) \\
\mathbf{O}_{2}(j)=f\left(X_{2}(j)\right) & (j=1, \cdots, S) \\
\mathbf{L}(j)=f\left(X_{3}(j)\right) & (j=1,2)
\end{array}
$$

The input and output function of various nodes is

$$
y=f(x)=\frac{1}{1+e^{-x}}
$$


So, $\frac{d y}{d x}=y(1-y)$, and $K_{i i}^{N}(W), K_{i j}^{N}(W)$ and the one-order partial derivatives to $W_{1}(i, j), W_{2}(i, j), W_{3}(i, j)$ can be calculated.

Replace above formulas into (9) and (3), $\Delta W$ can be obtained, and the NN decoupler is

$W=W+\Delta W$

Computer the objective function (2), when $E<\varepsilon$ (very small positive number), end the iteration process.

\section{Decoupling control simulation of total pressure and stock level}

By the method of the mechanism modeling or the model distinguish (Gao, 2003), the one-order inertial model about the total pressure and the stock level of the headbox can be simplified.

$\left[\begin{array}{l}P(s) \\ H(s)\end{array}\right]=\left[\begin{array}{cc}\frac{1.9}{36 s+1} & \frac{0.7}{27 s+1} \\ \frac{0.9}{36 s+1} & -\frac{1.5}{27 s+1}\end{array}\right]\left[\begin{array}{l}u_{F}(s) \\ u_{P}(s)\end{array}\right]$

The parameters of both controllers respectively are

The control total pressure of the stock inlet capacity adjustment, $P I: K_{p}=0.73, K_{I}=0.03$.

The control stock level of the air-cushioned pressure adjustment, $P I: K_{p}=-0.8, K_{I}=-0.043$.

Use above algorithm to train the NN decoupler, and when the amount of the hidden node is 8 , the learning step length is 0.001 and the training times are about 60000 , the proper decoupler $W$ can be obtained. Use $W$ in the decoupling control, the simulation Figure 5 can be obtained.

From Figure 5 (a), the influences between total pressure and the stock level influence are large before decoupling, because the fluctuation would enter into the stable state after a certain period because of the coupling function of the system in the initial period. Figure 5 (b) indicates that the system control ability increases after decoupling, the coupling function between the total pressure and the stock level is almost overcomes, and the time entering into the stable state is reduced.

\section{Conclusions}

The analysis in the article indicates that the control ability of the system increases obviously after the headbox uses the NN decoupling control system. The coupling function between the total pressure and the stock level is almost overcomes, and the time entering into the stable state is reduced. So the decoupler composed by the feed forward NN has better decoupling ability.

In actual application, when the sheet type changes, the character of the pulp will change largely and the work point will move. NN possesses the trainability, and the train of $\mathrm{NN}$ can be implemented in the upper computer which can make NN re-modified with manual instruction and transmit the system parameters to lower computers, so NN can be applied in the system decoupling to exert the trainability and the adaptability of the network. But the training time of the network is too long and the training process needs human participation, so the real-time character of $\mathrm{NN}$ will be reduced. Therefore, the better training algorithm or decoupling algorithm should be sought, and to further study the self-adaptive decoupling of NN will more make for the use and push of NN as the decoupler.

\section{References}

Gao, Juan. (2003). Artificial Neural Network Principle and Simulation Examples. Beijing: China Machine Press.

Li, Minghui \& Li, Yan. (2007). Decoupling and Time-delay Control Strategy of Basis Weight and Moisture Content Measurement in Papermaking. Transactions of China Pulp and Paper. No. 22(4). P. 107-114.

Shen, Guojiang, Hu, Dan \& Sun, Youxian. (2004). Modeling and Control of Basis Weight and Moisture in Papermaking Process Using Artificial Neural Networks. Transactions of China Pulp and Paper. No. 19(1). P. 151-156.

Tang, Wei, Wang, Mengxiao \& Li, Minghui. (2005). The Summarize about the Control of Headbox of Paper Machine. Journal of Shaanxi University of Science and Technology (Natural Science Edition). No. 23(6). P. 129-134.

Tang, Wei, Wang, Mengxiao, Li, Minghui \& Xue, Huijian. (2006). The Advanced Control Strategies and Decoupling Algorithms of Headbox. Transactions of China Pulp and Paper. No. 21(1). P. 107-114.

Wang, Mengxiao, Sun, Yu \& Tang, Wei. (2003). Measurement and Control System and Engineering for the Pulp and Papermaking process. Beijing: Chemical Industry Press.

Zhong, Luo \& Rao, Wenbi. (2007). Artificial Neural Network and Its Integrated Application Technology. Beijing: Science Press. 


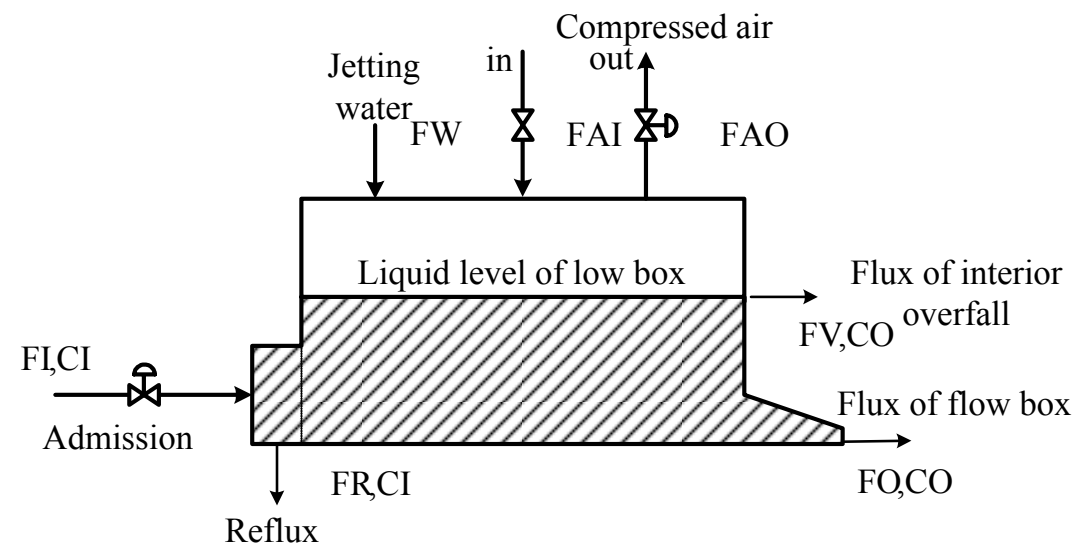

Figure 1. Sketch Map of Air-cushioned Type Headbox System

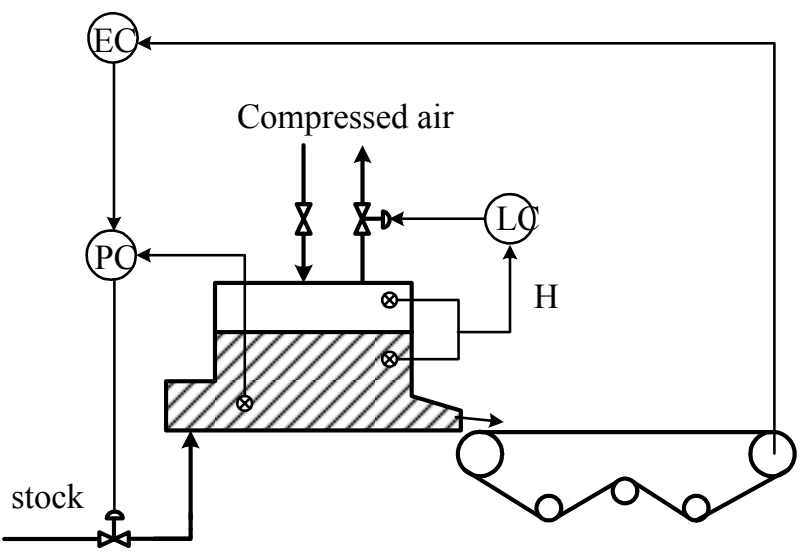

Figure 2. Adjustment Project of the Headbox

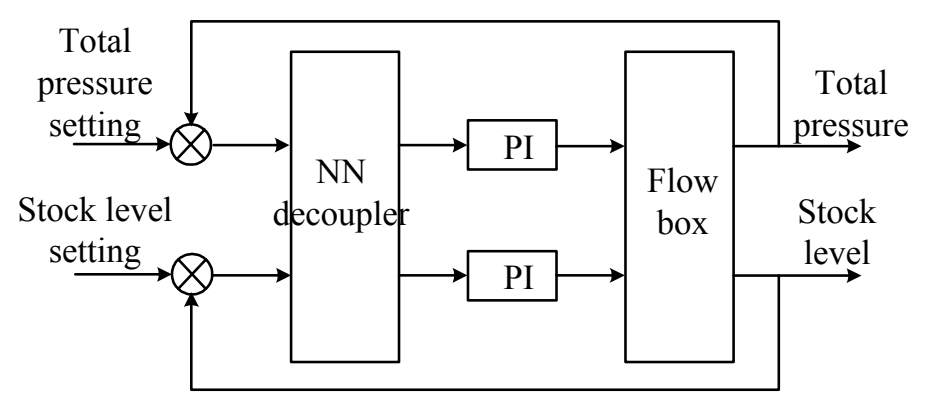

Figure 3. NN Decoupling Control Project

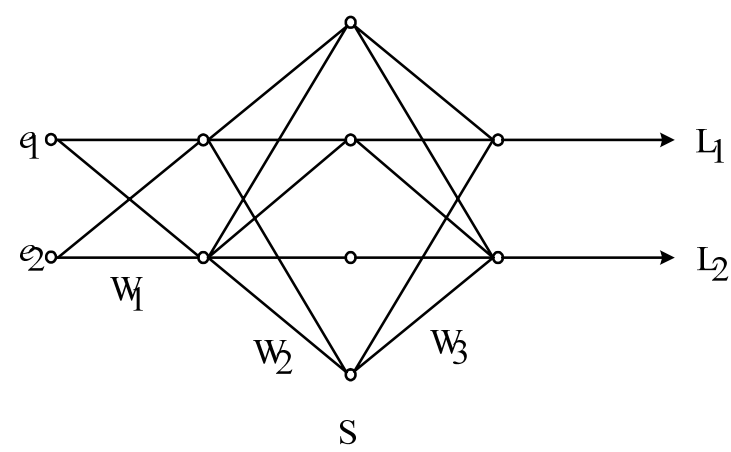

Figure 4. Three-layer NN Structure 

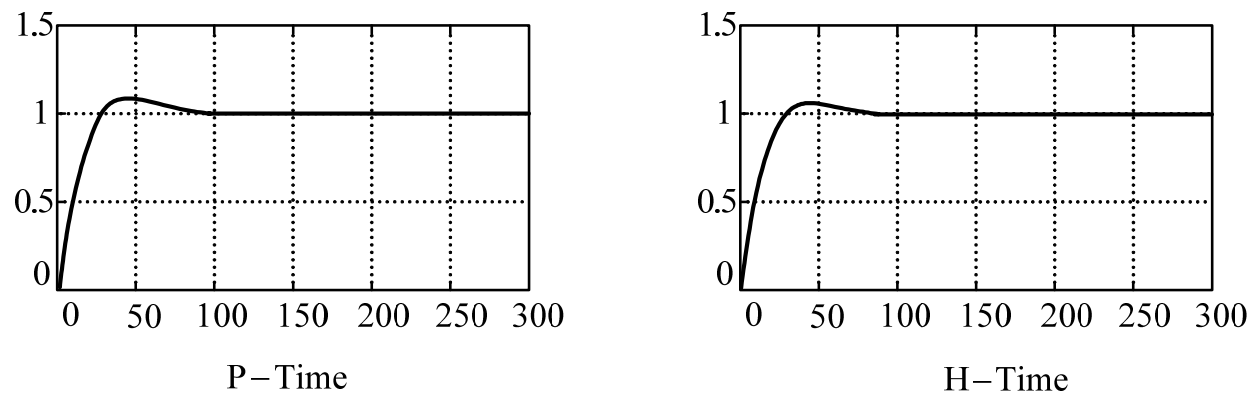

(a) Simulation of total pressure $(\mathrm{P})$ and Stock level $(\mathrm{H})$ before decoupling
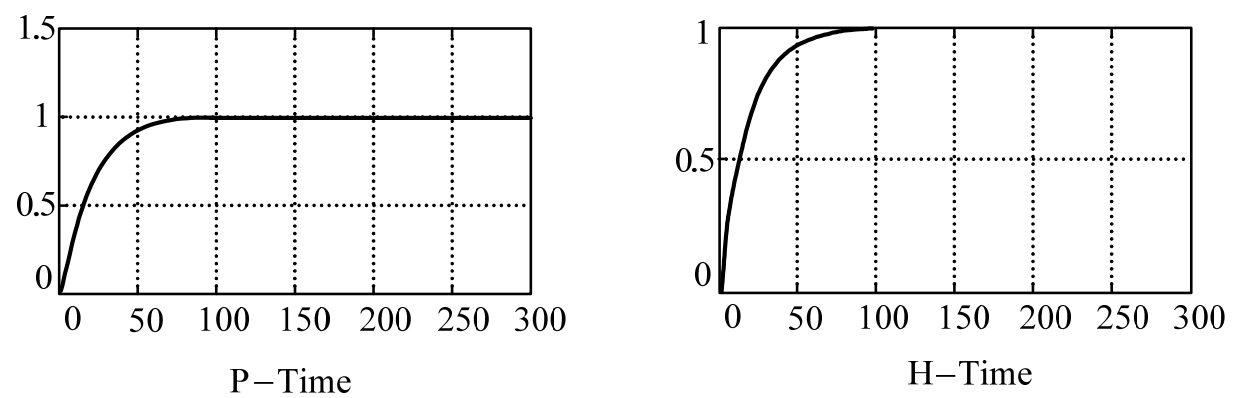

(b) Simulation of total pressure $(\mathrm{P})$ and Stock level $(\mathrm{H})$ after decoupling

Figure 5. Total Pressure and Stock Level Control System Simulation of the Headbox 